\title{
Applied Dynamic Programming for Optimization of Dynamical Systems
}

\section{Rush D. Robinett III}

Sandia National Laboratories

Albuquerque, New Mexico

\section{David G. Wilson}

Sandia National Laboratories

Albuquerque, New Mexico

\section{G. Richard Eisler}

Sandia National Laboratories

Albuquerque, New Mexico

\section{John E. Hurtado}

Texas A\&M University

College Station, Texas

\section{siam}




\section{Contents}

List of Figures $\quad$ ix

List of Tables $\quad$ xiii

$\begin{array}{ll}\text { Preface } & \text { xv }\end{array}$

1 Introduction 1

1.1 Key Role of Dynamic Programming in Control Design . . . . . . . . . 1

1.2 Illustrative Applications . . . . . . . . . . . . . . . . 2

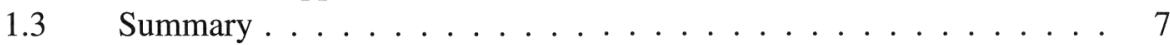

2 Constrained Optimization 9

$2.1 \quad$ Introduction . . . . . . . . . . . . . . . . 9

2.1.1 Nomenclature for Iterative Optimization Methods . . . . 9

2.2 Unconstrained Optimization-The Descent Property . . . . . . . . . . 10

2.3 Including the Constraints . . . . . . . . . . . . . . . . . 13

2.3.1 Equality Constraints . . . . . . . . . . . 13

2.3.2 Inequality Constraints and the Karush-Kuhn-Tucker

Conditions ................... . 14

2.4 Improving the Search with Second-Order Methods . . . . . . . . . . . 16

2.4.1 Newton's Method . . . . . . . . . . . 16

2.4.2 Updating the Hessian for Curvature Input . . . . . . . . . 17

2.5 The Quadratic Programming Problem . . . . . . . . . . . . . . . . . . 19

2.5.1 Solving the Linear System of Unknowns . . . . . . . . 19

2.5.2 A Merit Function for One-Dimensional Search . . . . . . 21

2.5.3 A Measure of Convergence and the Recursive
Quadratic Programming Algorithm . . . . . . . . . 21

2.6 Applications of Constrained Minimization . . . . . . . . . . . . 23

2.6.1 Implementation of the Recursive Quadratic

Programming Algorithm . . . . . . . . . . . . . 23

2.6.2 Optimizing Efficiencies in the Laser Welding Process . . 24

2.6.3 Maximum Terminal Velocity for Hypersonic Standoff

Missiles ............... . . . 29 
2.6.4 Optimal Slew Maneuvers for Space Telescope Attitude

Control . . . . . . . . . . . . . . . 37

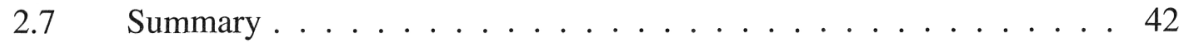

3 Introduction to Dynamic Programming 45

3.1 Introduction . . . . . . . . . . . . . . . . . 45

3.2 Discrete Dynamic Programming . . . . . . . . . . . . 45

3.2.1 Application of Discrete Dynamic Programming to Data

Smoothing . . . . . . . . . . . . . 47

3.2.2 Application of Discrete Dynamic Programming to

Discrete-Time Optimal Control Problems . . . . . . . . . 50 50

3.2.3 Implementation Details . . . . . . . . . . . 52

3.3 A Nonlinear Optimal Control Problem with Constraints . . . . . . . 57

3.4 Summary . . . . . . . . . . . . . . . . . 65

4 Advanced Dynamic Programming 67

4.1 Introduction . . . . . . . . . . . . . . . . 67

4.2 A Dynamic Programming Approach to Rocket Guidance Problems . . 67

4.2.1 Physical Model . . . . . . . . . . . . . 69

4.2.2 Guidance Problem . . . . . . . . . . . . . . 70

4.2.3 Transformation of the Guidance Problem . . . . . . . 72

4.2.4 Dynamic Programming Solution ... . . . . . . 74

4.2.5 Numerical Simulation Results . . . . . . . . . . . 75

4.3 Sequential Quadratic Programming Implementations for

Equality-Constrained Optimal Control . . . . . . . . . . . . . . . 77

4.3.1 Fixed Final Time Problems . . . . . . . . . . . . 77

4.3.2 Derivation of Recursive Equations . . . . . . . . . 81

4.3.3 Constraint Equations . . . . . . . . . . . . 83

4.3.4 Algorithm for P1 . . . . . . . . . . . . . . 84

4.3.5 Free Final Time Problems . . . . . . . . . . . . 85

4.3.6 Algorithm for P2 . . . . . . . . . . . . 86

$4.3 .7 \quad$ Practical Issues . . . . . . . . . . . . . 86

4.3.8 Example Problems . . . . . . . . . . . . 87

4.4 A Dynamic Programming Method for Constrained Optimal Control . . 96

4.4.1 Problem Formulation . . . . . . . . . . . . . 97

4.4.2 Sequential Quadratic Programming Subproblem

Formulation . . . . . . . . . . . . . . . 98

4.4.3 Interior Point Methods . . . . . . . . . . . . . 100

4.4.4 Equivalent Problem Formulation . . . . . . . . . . . 102

4.4.5 Details of Algorithm . . . . . . . . . . . 105

4.4.6 Example Problems . . . . . . . . . . . . . . . . 108

4.5 Summary . . . . . . . . . . . . . . . . 114

$5 \quad$ Applied Case Studies 117

$5.1 \quad$ Introduction . . . . . . . . . . . . . . . . . 117

5.2 Case Study 1: Rotary Jib Crane . . . . . . . . . . . . . . . . . . 118 
$5.2 .1 \quad$ Introduction $\ldots \ldots \ldots \ldots 118$

5.2.2 Model Development . . . . . . . . . . . . . . 119

5.2.3 Optimization Designs . . . . . . . . . . . . . 124

$5.2 .4 \quad$ Implementation Issues . . . . . . . . . . . . . . 125

5.2.5 Numerical Simulation Results . . . . . . . . . . . 126

5.2.6 Case Study 1 Discussion (Summary/Conclusions) . . . . 132

5.3 Case Study 2: Slewing Flexible Link . . . . . . . . . . . . . . . . 134

5.3.1 Introduction . . . . . . . . . . . . . 134

5.3.2 Model Development . . . . . . . . . . . 136

5.3.3 Optimization Feedforward Command Design . . . . . . . 142

5.3.4 Implementation Issues . . . . . . . . . . . . . . . . 145

5.3.5 Numerical Simulation Results . . . . . . . . . . . . 146

5.3.6 Case Study 2 Discussion (Summary/Conclusions) . . . 150

5.4 Case Study 3: Flexible Planar Arm . . . . . . . . . . . . . . 151

$5.4 .1 \quad$ Introduction . . . . . . . . . . . . 151

5.4.2 Model Development . . . . . . . . . . . 152

5.4.3 Dynamic Programming Optimization Design . . . . . . 162

5.4.4 Implementation Issues . . . . . . . . . . . . . 163

5.4.5 Numerical Simulation Results . . . . . . . . . . . 163

5.4.6 Case Study 3 Discussion (Summary/Conclusions) . . . . 167

5.5 Case Study 4: Minimum-Time Maneuvers of Rigid Systems . . . . . . 170

5.5 .1 Introduction . . . . . . . . . . . 170

5.5.2 The Classic Double Integrator Example . . . . . . . . . . 171

5.5.3 Three-Axis Maneuvers of a Symmetric Spacecraft . . . . 173

5.5.4 Case Study 4 Discussion (Summary/Conclusions) . . . . 177

5.6 Case Study 5: Maximum-Radius Orbit Transfer . . . . . . . . . . . 177

5.6.1 Introduction . . . . . . . . . . . . . 177

$5.6 .2 \quad$ Dynamical Model . . . . . . . . . . 178

5.6.3 Solution Using Dynamic Programming/Interior Point . . 178

5.6.4 Case Study 5 Discussion (Summary/Conclusions) . . . . 179

5.7 Case Study 6: PUMA 560 Industrial Manipulator . . . . . . . . . . 180

5.7 .1 Introduction . . . . . . . . . . . . 180

5.7.2 Model Development . . . . . . . . . . . . 180

5.7.3 Dynamic Programming Optimization Design . . . . . . . 184

$5.7 .4 \quad$ Implementation Issues . . . . . . . . . . . . . . . 185

5.7.5 Numerical Simulation Results . . . . . . . . . . 186

5.7.6 Case Study 6 Discussion (Summary/Conclusions) . . . . 189

$5.8 \quad$ Summary . . . . . . . . . . . . . . . . . . . . 189

A Mathematical Supplement 191

A.1 Determination of the State Transition Matrices . . . . . . . . . . . . 191

A.2 Example 4.3 Numerical Approach . . . . . . . . . . . . . . . . 192

A.3 Modified Dynamic Programming Algorithm . . . . . . . . . . . . . 194

B Applied Case Studies-MATLAB Software Addendum 197

B.1 Case Study 1 Driver Functions and Script Files . . . . . . . . . . . . 197 
B.1.1 Rotary Jib Crane Numerical Simulations Code . . . . . . 197

B.1.2 Dynamic Programming/Interior-Point Driver, Cost,

Equality, Inequality, and Dynamics Code . . . . . . . . . 202

B.2 Case Study 2 Driver Functions and Script Files . . . . . . . . . . . . . 205

B.2.1 Slewing Flexible Link Numerical Simulations Code . . . 205

B.2.2 Dynamic Programming/Interior-Point Driver, Cost,

Equality, Inequality, and Dynamics Code . . . . . . . . 209

B.2.3 fmincon Driver, Cost, Constraints, and Dynamics Code . 215

B.3 Case Study 3 Driver Functions and Script Files . . . . . . . . . . . . 219

B.3.1 Dynamic Programming/Interior-Point Driver, Cost,

Equality, Inequality, and Dynamics Code . . . . . . . . . 219

B.4 Case Study 4 Driver Functions and Script Files . . . . . . . . . . . . 225

B.4.1 Dynamic Programming/Interior-Point Driver, Cost,

Equality, Inequality, and Dynamics Code . . . . . . . . . 225

B.5 Case Study 5 Driver Functions and Script Files . . . . . . . . . . . . 229

B.5.1 Dynamic Programming/Interior-Point Driver, Cost,

Equality, Inequality, and Dynamics Code . . . . . . . . . 229

B.6 Case Study 6 Driver Functions and Script Files . . . . . . . . . . . . . 233

B.6.1 Dynamic Programming/Interior-Point Driver, Cost,

Equality, Inequality, and Dynamics Code . . . . . . . . . 233

B.6.2 PUMA560 Dynamic Equations of Motion C-Code . . . . 239

B.7 Discrete Dynamic Programming Standalone Code-Discrete-Time

Optimal Control Problem . . . . . . . . . . . . . . . . . . 246

Bibliography

Index 